\title{
Reliability prediction of RFID passive tags power supply systems based on RTD under given operating conditions
}

\author{
Mstislav Makeev ${ }^{1,2, *}$, Vladimir Sinyakin ${ }^{1}$, and Sergey Meshkov ${ }^{1}$ \\ ${ }^{1}$ Bauman Moscow State Technical University, 105005 Moscow, Russia \\ ${ }^{2}$ Peoples' Friendship University of Russia (RUDN University), Moscow, 117198 Moscow, Russia
}

\begin{abstract}
The technique for predicting RFID passive tag power supply system reliability, the failure of which is caused by the degradation of RTDs that are part of the tag rectifier, is developed. The calculation of the time to failure of the RFID passive tag power supply system by the criterion of the minimum permissible range is presented based on the developed technique.
\end{abstract}

\section{Introduction}

The current practice of designing of micro-and nanodevices includes systems engineering, circuit and engineering design, technological process preparation stages. The device structure is determined at the system engineering stage. The electrical circuit and circuit elements parameters that determine the functional parameters of the device $\boldsymbol{Y}$ (purpose indicators) are the results of circuit design stage. The design implementation of circuit solutions and design parameters $\boldsymbol{X}$, which define the required parameters $\boldsymbol{Y}$, are determined at the design stage. The technological route is developed, the parameters of technological operations are determined and the necessary technological equipment is designed at the technological process preparation (TPP) stage.

This methodology does not take into account the fact that the micro- and nanodevices design imply the use of group technology production methods (often planar), if the device construction elements are located on the same substrate. The high correlation of the devices parameters formed simultaneously within the common substrate during the structural elements production operations is a feature of group technologies. On the other hand, the location of similar structural elements on a common substrate is the reason for the high correlation of aging and degradation processes occurring under the influence of destabilizing factors during device operation. This leads to the fact that the existing linear design procedure does not allow to achieve devices line optimal technological efficiency and reliability indicators.

The incorporation of new electronic components is needed for enhancing functionality and improving the parameters of modern radio electronics. The resonant-tunneling diode (RTD) based on multilayer nanoscale semiconductor $\mathrm{A}_{3} \mathrm{~B}_{5}$ heterostructures is a promising

* Corresponding author: mc.stiv@gmail.com 
component [1-6]. The prospects of the RTD for modern radio electronics are caused by the following circumstances:

-RTDs limit frequency spreads up to units of THz, which makes the RTD a promising device for SHF, EHF, and THF electronics.

-RTDs operates in the range of temperatures and other external factors necessary for technical applications.

- Technologies and the equipment for RTD production are available at enterprises producing heterostructural electronics devices in particular, monolithic integration circuits.

-It is possible to control the shape of the current-voltage characteristic (CVC) and create a diode with the optimum CVC shape for the specific type of nonlinear conversion by changing the heterostructure layers parameters (thickness, chemical composition). The set of possible nonlinear transformations using RTD is very wide: generation of radio signals, frequency modulation, radio frequency mixing, amplitude detection, rectification, frequency labels grid generation, etc. The advantages of RTDs application in SHF mixers, as well as in the low-power RF rectifiers, are shown in [7-11].

At the same time, when incorporate a new component, data on its reliability are needed. This work is devoted to the development of a technique for reliability predicting of RFID passive tags power supply systems based on RTD under given operating conditions.

\section{RFID passive tag power supply system}

Rectifiers of low-power RF signals have found wide application in radio frequency identification (RFID) technology with passive tags $[12,13]$. This technology is widely used in the trading system for the accounting of goods, in the logistics of transport companies, in enterprises for recording the working time of employees, in medicine and other spheres.

The RFID system consists of a reader and one or more tags that contain information about the objects carrying them. The reader sends using the radio channel a request to the tag, which receives it, processes it and responds to the request. The reader receives information from the tag and sends it to the communication channel with the data collection unit.

The power of the passive tag is provided by the electromagnetic energy of the reader. $\mathrm{RF}$ signal of the reader is received by the tag's antenna and is converted into AC, which is fed to the input of the RF signal rectifier. The rectifier converts it into a direct current, used to power the functional units of the tag. The range of RFID systems with passive tags is determined by the sensitivity of the tag, i.e. the minimum signal power at the antenna input, which is necessary to form a tag supply DC voltage sufficient for its normal operation.

The sensitivity of the tag is determined by the ability of the antenna and the rectifier to convert the electromagnetic radiation of the reader into DC. The complex of the tag antenna and the RF signal rectifier in the literature is called as rectenna (rectifying antenna). It is characterized by an efficiency factor, which is the product of the antenna-rectifier matching factor $K_{\mathrm{Z}}$ and the efficiency of the rectifier $\eta_{\mathrm{R}}[10]$ :

$$
K_{\mathrm{E}}=K_{\mathrm{Z}} \cdot \eta_{\mathrm{R}}
$$

The matching factor shows the part of the RF signal power from the antenna obtained by the rectifier, and is determined by the formula: 


$$
K_{\mathrm{Z}}=\frac{4 \cdot R_{\mathrm{R}} \cdot R_{\mathrm{A}}}{\left|Z_{\mathrm{R}}+Z_{\mathrm{A}}\right|^{2}},
$$

where $Z_{\mathrm{A}}=R_{\mathrm{A}}+j X_{\mathrm{A}}$ is antenna impedance; $Z_{\mathrm{R}}=R_{\mathrm{R}}+j X_{\mathrm{R}}$ is rectifier impedance.

The rectifier conversion efficiency is the ratio of the power consumed by the tag functional units $P_{\mathrm{DC}}$ to the RF power at the input of the rectifier $P_{\mathrm{RF}}$ :

$$
\eta_{\mathrm{R}}=\frac{P_{\mathrm{DC}}}{P_{\mathrm{RF}}} .
$$

The rectifier efficiency is determined firstly by the design of the rectifier, and secondly by the form of the rectifier nonlinear element IV-curve. Schottky barrier diodes (SBD) or diode-mounted field-effect transistors are usually used as nonlinear elements in a rectifier. However, these elements have a significant drawback associated with the presence of a threshold voltage in their IV-curve. RTD with an IV-curve optimized for rectification of weak RF signals are free of this drawback [8-10]. The authors' research has shown that by using such RTDs in the RF signal rectifier it is possible to increase the sensitivity of the passive tag by $3 \ldots 5$ times compared to the tag based on the SBD.

Using formulas (1)-(3) as well as the formula of an ideal radio transmission [14] we can determine the range of the RFID system as

$$
r=\sqrt{\frac{P_{\mathrm{EIRP}} \cdot A_{\mathrm{E}} \cdot K_{\mathrm{E}}}{4 \pi P_{\mathrm{DC}}}},
$$

where $P_{\text {EIRP }}$ is the equivalent isotropic radiated power of the reader antenna; $A_{\mathrm{E}}$ is the effective aperture of the tag antenna.

The effective aperture of the antenna is determined by the formula

$$
A_{\mathrm{E}}=\frac{\lambda^{2} G}{4 \pi},
$$

where $\lambda$ is the wavelength of the reader RF signal; $G$ is the antenna gain.

\section{The technique of reliability prediction of RFID passive tags power supply systems based on RTD under given operating conditions}

When incorporating a new component, data on its reliability are needed. The most valid and complete reliability indices of radio electronic systems (RESs) provide operation results. However, this requires a large time. Traditional test methods in many cases do not make it possible to confirm the specified level of reliability of RES due to the presence of hidden defects, as well as in view of the fact that the intentionally entered acceleration of degradation processes during the tests does not always lead to failure.

The RTD contains a resonant-tunneling structure (RTS, set of AlAs/GaAs layers), nearcontact regions (Si-alloyed GaAs layers), and ohmic contacts (Fig. 1). The RTD I-V characteristic shape is determined by the RTS structure, near-contact region resistance $R_{n-n+}$ and ohmic contact resistance $R_{C}$. The RTS consists of layers up to several atomic layers thick, which cause high sensitivity of the RTD I-V characteristic to changes in its parameters. Degradation processes, i.e., the interlayer diffusion of Al in the RTS and $\mathrm{Si}$ in 
the near-contact regions, lead to changes in the potential barrier and well shape and, as a result, to a change in the charge-transport conditions in the structure. The diffusive spreading of ohmic contacts caused to an increase of their contact resistance. These processes in turn cause drift of the RTD I-V characteristic and degradation of the purpose indicators of rectifier based on RTDs.

Diffusion processes in the RTS and ohmic contacts occur simultaneously, but have different rates. Therefore, their contributions to the change in the RTD I-V characteristic are different. We will distinguish the mathematical model of the RTS and near-contact regions degradation and the mathematical model of the ohmic contacts degradation.

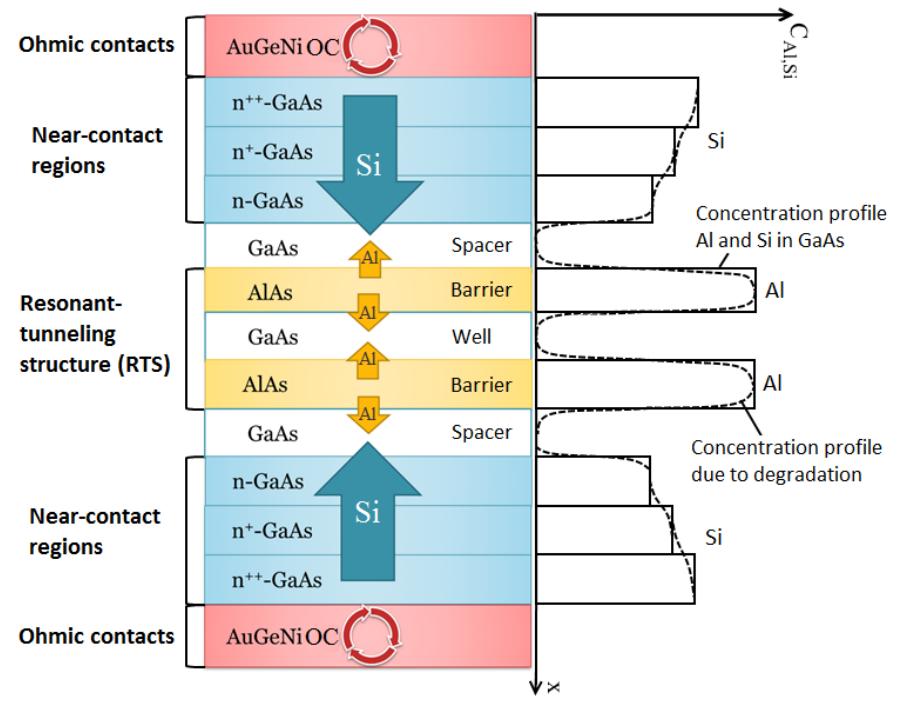

Fig. 1. Structure and possible degradation phenomena in RTD.

The semiconductor resonant-tunneling heterostructure degradation occurs as a result of diffusion of $\mathrm{Al}$ and $\mathrm{Si}$ in the RTS and near-contact regions. RTS and near-contact regions degradation mathematical model is based on Fick's law. The diffusion coefficients of Al and $\mathrm{Si}$ in GaAs are determined [14-17]:

$$
D_{\mathrm{Al}, \mathrm{Si}}(n)=D_{0} \cdot e^{\left(\frac{-E_{a}}{k T}\right)} \cdot\left(\frac{n}{n_{i}}\right)^{3} .
$$

where $D_{\mathrm{Al}, \mathrm{Si}}(n)$ is $\mathrm{Al}$ and $\mathrm{Si}$ diffusion coefficient in $\mathrm{n}$-doping $\mathrm{GaAs} ; D_{0}$ is the preexponential factor in units of $\mathrm{cm}^{2} / \mathrm{s} ; E_{a}$ is the activation energy in units of $\mathrm{eV} ; n$ и $n_{i}$ are the electrons concentration in the conduction band and the intrinsic carrier concentration in units of $\mathrm{cm}^{-3} ; T$ - temperature, $\mathrm{K} ; k$ - Boltzmann constant $\left(\mathrm{k}=8,61710^{-5} \mathrm{eV} \cdot \mathrm{K}^{-1}\right)$.

Ohmic contacts degradation mathematical model makes it possible to obtain the kinetics of RTD specific contact resistance as a function of time and temperature and is described by equation [18-20]:

$$
\rho_{C}=\rho_{C 0}+\chi \cdot \exp \left(-\frac{E_{a}}{2 k T}\right) \cdot \sqrt{t}
$$


where $\rho_{C 0}$ is initial ohmic contact resistivity (after the production) in units of $\mathrm{Ohm} \cdot \mathrm{cm}^{2} ; \chi$ is a coefficient, depending on the design and technology of ohmic contacts in units of $\left(\mathrm{Ohm} \cdot \mathrm{cm}^{2}\right) / \mathrm{s}^{1 / 2}, E_{a}$ is the activation energy in units of $\mathrm{eV} ; t$ is a time in units of $\mathrm{s}$.

RTS current transport mathematical model is used to calculate the RTS I-V characteristic. It is based on Tsu-Esaki tunneling current formula [21]:

$$
J(E, U)=C \int_{0}^{\infty} E_{F} \cdot F(E, U) \cdot T(E, U) d E .
$$

where $J$ is current density across the layers in units of $\mathrm{A} / \mathrm{cm}^{2} ; U$ is the voltage applied to the studied structure in units of $\mathrm{V} ; C$ is the constant equal to $2 \cdot 10^{9} \mathrm{~A} /\left(\mathrm{cm}^{2} \cdot \mathrm{eV}^{2}\right) ; E$ is the kinetic energy of the motion of electrons in the conduction band across the RTS layers in units of $\mathrm{eV} ; E_{F}$ is the Fermi level in units of $\mathrm{eV} ; F(E, U)$ is the Fermi subintegral function, characterizes the energy density of the electron distribution; $T(E, U)$ is the heterostructure transmission coefficient, $T(E, U)$ is calculated by the transfer matrix method.

Application of the RTS and near-contact regions degradation, ohmic contacts degradation and RTS current transport mathematical models makes it possible to obtain the kinetics of the RTD I-V characteristics under the influence of operational factors.

In this work, the reliability of RFID passive tags power supply systems based on RTD are based on gradual failure model. As a criterion for the rectenna failure, RFID system range reduction up to a minimum permissible value $r_{\min }$ is assumed. It is supposed that the system range reduction is due to a rectenna sensitivity decrease, which is caused by the RTDs degradation. The RTD degradation at operation leads to a change in its I-V characteristic. As a result, the rectenna efficiency factor and RFID system purpose indicators decreases. In particular, RFID system range decreases. The influence of other analog and digital tag elements on the power supply system operation is not taken into account. The failures of the other rectenna elements are considered as sudden and are caused to rectenna catastrophic failures. The latter are not taken into account in the gradual failure model.

Therefore, the reliability model of the RFID passive tags power supply system based on the RTD includes:

a) The passive tags power supply system model. Its output parameter is the RFID system range;

b) RTD degradation model, which consist of RTS and near-contact regions degradation, ohmic contacts degradation and RTS current transport mathematical models.

As the acceleration factor of RTD degradation processes an elevated temperature is considered. Based on this, the technique of reliability prediction of the RFID passive tags power supply system based on RTDs was developed. Its algorithm is shown in Fig. 2. Briefly, the technique can be described as follows:

1. The initial value of the RFID system range $r_{0}$ is calculated using the passive tags power supply system based on RTD model and RTS current transport model described hereinbefore.

2. Passive tag operation temperature $T$ is defined.

3. Increment $\Delta t$ is added to the value of the operating time $t: t_{i+1}=t_{i}+\Delta t$.

4. RTD IV-curves are calculated for each value of $t_{i}$ using the RTD degradation models (6) and (7), and a new value of the RFID system range $r_{i}$ is calculated.

5. The new value of the RFID system range $r_{i}$ is compared with the $r_{\text {min }}$ system failure criterion. If $r>r_{\min }$, it is necessary to perform steps 3 and 4 . Otherwise, the calculated value $t_{i}$ can be taken as time to failure. 
The results of calculating the time to failure of the RFID passive tag power supply system at operating temperatures of $25^{\circ} \mathrm{C}$ and $85^{\circ} \mathrm{C}$ are shown in Fig. 2. As initial data for the calculation are accepted:

a) the RTS structure: GaAs spacer $(21 \AA) / \mathrm{Al}_{0,3} \mathrm{Ga}_{0,7} \mathrm{As}$ barrier $(21 \AA) / \mathrm{GaAs}$ well $(100 \AA) / \operatorname{AlAs}$ barrier $(42 \AA) / \mathrm{GaAs}$ spacer $(21 \AA)$;

b) RTD construction parameters:

- the lower ohmic contact area is $2490 \mu \mathrm{m}^{2}$;

- mesa area is $9 \mu \mathrm{m}^{2}$;

- the initial ohmic contact resistivity $\rho_{\mathrm{C} 0}=1 \cdot 10^{-6} \mathrm{Ohm} \cdot \mathrm{cm}^{2}$;

- the activation parameters of diffusion $\mathrm{Al}$ and $\mathrm{Si}$ in RTS and near-contact regions: $D_{0}=0.22 \mathrm{~cm}^{2} / \mathrm{s}, E_{a}=3,5 \mathrm{eV}$.

- ohmic contacts degradation specific coefficient $\chi=0,027\left(\mathrm{Ohm} \cdot \mathrm{cm}^{2}\right) / \mathrm{s}^{1 / 2}$; the activation energy $E_{a}$ of degradation phenomena of AuGeNi ohmic contacts equals $1 \mathrm{eV}$ [22].

c) rectenna construction parameters:

- $\quad$ number of diodes in the rectifier $N_{\mathrm{D}}=36$;

- $\quad$ antenna impedance $Z_{\mathrm{A}}=61 \mathrm{Ohm;}$

- $\quad$ antenna gain $G=1,66$.

d) load parameters:

- $\quad$ minimum load operating voltage $U_{\mathrm{L}}=1,5 \mathrm{~V}$;

- $\quad$ load operating current $I_{\mathrm{L}}=10 \mu \mathrm{A}$.

e) reader parameters:

- $\quad$ operating frequency $f_{0}=910 \mathrm{MHz}$;

- $\quad$ equivalent isotropic radiated power of the reader antenna $P_{\mathrm{EIRP}}=4 \mathrm{~W}$;
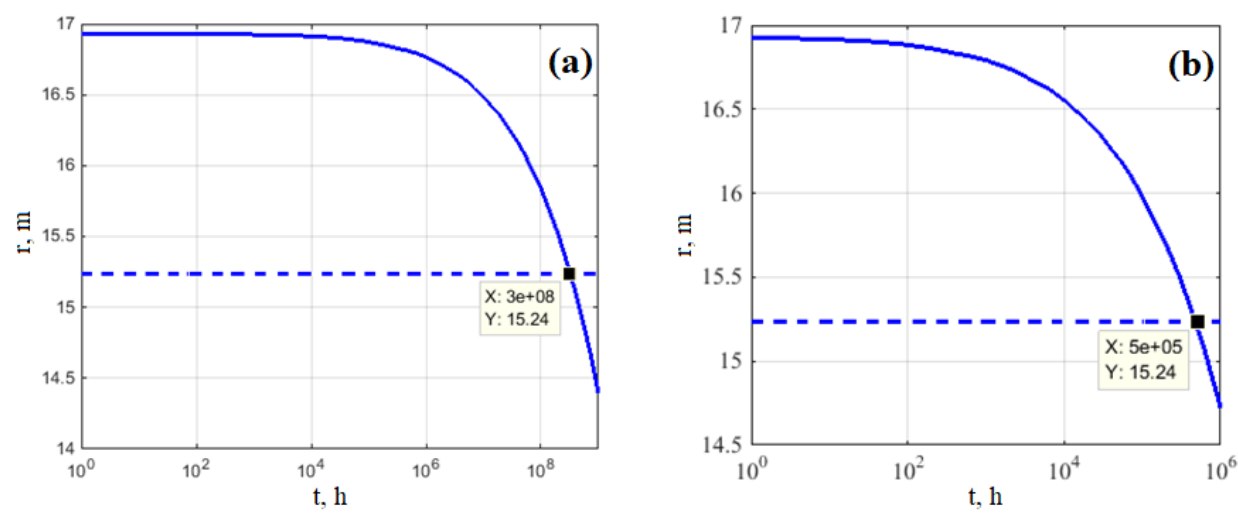

Fig. 2. The RFID system range as a function of time: (a) - $T=+25^{\circ} \mathrm{C}$, (b) $-T=+85^{\circ} \mathrm{C}$

The calculation showed that the time to failure according to the selected criterion is reduced from $3 \times 10^{8}$ hours to $5 \times 10^{5}$ hours with an increase in the operating temperature from $25^{\circ} \mathrm{C}$ to $85^{\circ} \mathrm{C}$.

\section{Conclusions}

In this paper, we considered a technique for predicting the RFID passive tag power supply system reliability, the failure of which is caused by the degradation of RTDs that are part of the tag rectifier. The technique allows to calculate the time to failure of the RFID passive tag power supply system by the criterion of the minimum permissible range. The chosen 
failure criterion (the reduction of the RFID system range below the critical value) does not limit the generality of the developed technique. If it is necessary to introduce another criterion of failure or several criteria in the technique, a power supply model with other output parameters can be used. RTS current transport model, RTS and near-contact regions degradation model and ohmic contacts degradation model will remain unchanged.

The results of the time to the failure calculation are comparable with the corresponding indicators of modern semiconductor devices. The developed technique can be used in the design of RFID systems with passive UHF and SHF tags to predict their reliability.

The reported study is funded by RFBR, according to the research project No. 16-37-60067 mol_a dk. The publication was prepared with the support of the "RUDN University Program 5-100".

\section{References}

1. H. Mizuta, T. Tanoue, High-speed and functional applications of resonant tunnelling diodes. In The Physics and Applications of Resonant Tunnelling Diodes (Cambridge university press, New York, 2006)

2. S. Suzuki, M. Asada, A. Teranishi, H. Sugiyama, H. Yokoyama, Appl. Phys. Lett. 97 242102 (2010)

3. M. Feiginov, C. Sydlo, O. Cojocari, P. Meissner, Appl. Phys. Lett. 99 233506-1-3 (2011)

4. K. Ishigaki et al., Electron. Lett., 48 (10) 582-583 (2012)

5. H. Kanaya, H. Shibayama H, R. Sogabe, S. Suzuki, M. Asada Applied Physics Express 5124101 (2012)

6. T. Maekawa, H. Kanaya, S. Suzuki, M. Asada, Applied Physics Express 9024101 (2016)

7. Y.A. Ivanov, S.A. Meshkov, I.A. Fedorenko et al, J. Commun. Technol. Electron 55 921-927 (2010)

8. Y.A. Ivanov, A.G. Gudkov, S.V. Agasieva, S.A. Meshkov, V.Yu. Sinyakin, M.O. Makeev, Microwave \& Telecommunication Technology (CriMiCo), 2014 24th Int. Crimean Conf. 1063-1064 (2014)

9. A.G. Gudkov, Y.A. Ivanov, S.A. Meshkov et al, Biomedical Engineering 49 98-101 (2015)

10. V.Y. Sinyakin, M.O. Makeev, S.A. Meshkov, J. Phys.: Conf. Ser. 741012160 (2016)

11. M.O. Makeev, S.A. Meshkov, V.Yu. Sinyakin, Yu.N. Razoumny, Adv. in Astronautical Sci. 161475 (2017)

12. K. Finkenzeller, RFID Handbook Fundamentals and Applications in Contactless Smart Cards and Identification (John Wiley and Sons Ltd, Chichester, 2010)

13. M. Kaur, M. Sandhu, N. Mohan, P.S. Sandhu, International Journal of Computer and Electrical Engineering 3 (1) 1793-8163 (2011)

14. ,D.M. Sazonov, Antenny i ustroistva SVCH (Vyssh. shk., Moscow, 1988)

15. M.O. Makeev, Y.A. Ivanov, S.A. Meshkov, Semiconductors 50 83-88 (2016)

16. M.O. Makeev, S.A. Meshkov, Y.A. Ivanov, Key Engineering Materials 724 48-52 (2017)

17. M.O. Makeev, Y.A. Ivanov, V.Yu. Sinyakin, S.A. Meshkov, S.V. Agasieva, V.D. Shashurin, Microwave \& Telecommunication Technology (CriMiCo), 2014 24th Int. Crimean Conf. 754-755 (2014) 
18. M.O. Makeev, S.A. Meshkov, AIP Conference Proceedings 1858020001 (2017)

19. M.O. Makeev, Y.A. Ivanov, S.A. Meshkov et al, 2015 5th International Workshop on Computer Science and Engineering: Information Processing and Control Engineering, WCSE 2015-IPCE. 260-265 (2015)

20. M.O. Makeev, S.A. Meshkov, V.Yu. Sinyakin, J. Phys.: Conf. Ser. 917092004 (2017)

21. R. Tsu, L. Esaki, Appl. Phys. Lett. 22 562-564 (1973)

22. V.A. Vashchenko, V.F. Sinkevitch Physical limitations of semiconductor devices. (Springer, Berlin, 2008) 